\title{
Apoio contínuo na assistência ao parto para redução das cirurgias cesarianas: síntese de evidências para políticas
}

\author{
Continuous support during childbirth to reduce cesarean sections: \\ evidence brief for policy
}

Cintia de Freitas Oliveira (https://orcid.org/0000-0001-6331-6510) ${ }^{1}$

Maritsa Carla de Bortoli (https://orcid.org/0000-0001-8236-7233) ${ }^{1}$

Cecilia Setti (https://orcid.org/0000-0003-2936-4759) ${ }^{1}$

Cézar Donizetti Luquine Júnior (https://orcid.org/0000-0002-5038-6808) ${ }^{1}$

Tereza Setsuko Toma (https://orcid.org/0000-0001-9531-9951) ${ }^{1}$

${ }^{1}$ Núcleo de Evidências, Centro de Tecnologias de Saúde para o SUS-SP, Instituto de Saúde da Secretaria de Estado da Saúde de São Paulo. R. Santo Antônio 590, Bela Vista. 01314-000 São Paulo SP Brasil. cintiaf15@gmail.com
Abstract Cesarean section rates have increased globally in the last 30 years, representing more than $56 \%$ of total births in Brazil. Considering the impacts of elective cesarean sections in maternal and child health, this review aimed to present an option to reduce these rates. We used the SUPPORT tools to summarize the best evidence. We included systematic reviews published in English, Portuguese, or Spanish, which focused on non-clinical interventions. Continuous support during labor and birth was described in four systematic reviews as an option to reduce cesarean rates (down to -51\%). It also decreased unnecessary clinical interventions during labor, the $d u$ ration of labor, and the likelihood of delivering a baby with a low Apgar score at five minutes. Moreover, continuous support improved breastfeeding, postpartum recovery of women during the puerperium period, and increased women's satisfaction with the birth experience and the possibility of spontaneous vaginal deliveries. The continuous support was beneficial for women and newborns. The intervention fosters respect, safety, and quality during birth, besides reducing hospital costs. Therefore, it is an effective strategy that should be adopted in hospitals and other health services to improve women's access to their rights. Key words Cesarean section, Natural childbirth, Evidence-informed policy, Policy-making, Review
Resumo As taxas mundiais de cirurgia cesariana têm crescido há 30 anos, correspondendo a mais de 56\% dos nascimentos no Brasil. Considerando os agravos ocasionados por cesáreas eletivas na saúde materna e infantil, esta revisão buscou apresentar uma estratégia para a redução dessas taxas. Para realizar a sintese das melhores evidências, adotou-se a metodologia SUPPORT. Foram incluídas revisões sistemáticas publicadas em inglês, português ou espanhol sobre intervenções não clinicas. As buscas resultaram em quatro estudos, que em metanálise associaram o apoio contínuo durante o parto à redução de $25 \%$ a $51 \%$ nas taxas de cesárea. Igualmente, o apoio contínuo reduziu a duração e as intervenções clinicas desnecessárias durante o trabalho de parto e a probabilidade de bebê com baixo escore de Apgar aos cinco minutos. Além disso, proporcionou melhoria na amamentação e na recuperação da mulher, aumento da satisfação com o processo de nascimento e maiores chances de parto vaginal espontâneo. $O$ apoio contínuo foi benéfico para a gestante $e$ o recém-nascido. A intervenção garante respeito, segurança e qualidade durante o parto, bem como reduz custos hospitalares. Portanto, é uma estratégia efetiva que deve ser adotada nos serviços de saúde para ampliar o acesso das mulheres aos seus direitos.

Palavras-chave Cesárea, Parto normal, Política informada por evidências, Formulação de politicas, Revisão 


\section{Introdução}

O aumento nas taxas de cesariana preocupa cada vez mais pesquisadores, formuladores de políticas, profissionais de saúde e a sociedade civil, uma vez que a cirurgia está relacionada a desfechos negativos de curto e longo prazo, tanto para as mulheres quanto para os recém-nascidos. $\mathrm{O}$ crescimento na morbimortalidade materna, na prematuridade e na chance de óbito fetal e anormalidades placentárias em gestações futuras são exemplos que podem ser citados ${ }^{1}$. Além disso, a cesariana impacta negativamente a amamentação ${ }^{2}$ e o desenvolvimento do sistema imunológico e da microbiota do neonato, aumentando as chances de asma e alergias no futuro ${ }^{1}$.

Desde 1985, a Organização Mundial da Saúde (OMS) estabelece que a proporção de cesáreas deve representar entre $10 \%$ e $15 \%$ do total de nascimentos ${ }^{3}$, uma vez que valores maiores dificilmente se justificam do ponto de vista clínico ${ }^{4}$. Apesar disso, nos últimos 15 anos, as taxas mundiais de cesariana dobraram, chegando a $21 \%$ do total de nascimentos, e continuam a crescer cerca de $4 \%$ ao ano 5 .

O Brasil é o segundo país em realização de cesarianas, com taxas que passaram de $15 \% \mathrm{em}$ 1970 para $56 \%$ em 2016, ficando atrás apenas da República Dominicana $(59 \%)^{6,7}$. Ainda em relação ao cenário brasileiro, vale destacar a discrepância na proporção de cesarianas realizadas em serviços públicos em relação às da saúde suplementar. Em 2014, 87,7\% dos nascimentos no setor privado foram por via cirúrgica, em comparação a $42,9 \%$ no setor público ${ }^{8}$. Nas diferentes regiões do país, as cesarianas têm aumentado, proporcionalmente, de acordo com a cobertura dos planos de saúde. No entanto, em ambos os setores, aproximadamente $50 \%$ ocorrem de forma eletiva, com agendamento prévio $^{10}$.

As dificuldades brasileiras para reduzir as taxas de cesárea ressaltam a complexidade do problema, que tem origens em fontes como: a necessidade de lucro ou a falta de infraestrutura apropriada para atenção ao parto nos serviços de saúde; o acesso à cesárea eletiva como um bem de consumo; a maior comodidade da cirurgia para médicos e planos de saúde; e formação inadequada de profissionais de saúde ${ }^{11-13}$.

Sabe-se, nesse contexto, que a situação socioeconômica da gestante também impacta enormemente a decisão sobre a cesárea. Em hospitais privados, acessados por quem tem maior poder aquisitivo, prioriza-se a manutenção de lucros, enquanto a falta de leitos ou a insuficiência de recursos humanos é a justificativa utilizada em serviços públicos ${ }^{11}$.

No intuito de melhorar o cuidado materno e infantil no país, o que evidentemente inclui a redução das cesarianas sem indicação clínica, diversas estratégias têm sido implementadas desde a década de 1980. Entre elas, o Programa de Humanização no Pré-natal e Nascimento (2000), a Política Nacional de Atenção Integral à Saúde da Mulher (2004), a ampliação e revisão da Iniciativa Hospital Amigo da Criança (2006), a Rede Cegonha (2011), as Resoluções Normativas $n^{\circ}$ 368 e 398 da Agência Nacional de Saúde Suplementar - ANS (2015 e 2016), a Diretriz de Atenção Integral à Gestante: a operação Cesariana (2015), as Diretrizes Nacionais de Assistência ao Parto Normal (2017), as duas últimas formuladas pela Comissão Nacional de Incorporação de Tecnologias no SUS - CONITEC, o Projeto Parto Adequado (2016) e o Projeto Aprimoramento e Inovação no Cuidado e Ensino em Obstetrícia e Neonatologia - APICE ON (2017) ${ }^{14}$.

Embora alguns avanços sejam observados na assistência ao parto, as taxas de cesariana continuam a ser um problema de alta prioridade no Brasil. Dessa forma, quais intervenções são apontadas pela literatura mundial como eficazes para reduzir as taxas de cesarianas desnecessárias? Para responder a essa questão, realizamos uma síntese de evidências para políticas de saúde, que identificou cinco opções para reduzir as taxas de cesariana, a partir de revisões sistemáticas ${ }^{14}$ : intervenções combinadas, com foco em profissionais de saúde e na gestão dos serviços e sistemas de saúde; preparo da mulher para o parto normal durante o pré-natal; acesso a diferentes ambientes para o parto; apoio ao parto vaginal após cesárea prévia; e apoio contínuo às mulheres durante o trabalho de parto e parto.

Neste artigo, o objetivo é detalhar a opção de oferta de apoio contínuo, que pode ser definido como a presença ininterrupta de uma pessoa com a parturiente durante todo o trabalho de parto e parto, a fim de oferecer conforto emocional, medidas para alívio da dor, informações sobre a progressão do trabalho de parto e também favorecer a articulação dos desejos da mulher com as práticas da equipe de saúde ${ }^{15}$. Da mesma forma que a redução das cirurgias cesarianas, o apoio contínuo é uma das boas práticas de assistência ao parto recomendadas pela OMS há mais de 30 anos ${ }^{16}$. No entanto, o acesso a essa prática ainda não foi garantido a todas as gestantes, parturientes e puérperas, o que se mostrou um desafio ainda maior frente à pandemia da covid-19, não 
apenas no Brasil, mas em outras localidades ${ }^{17,18}$. $\mathrm{O}$ artigo apresenta também elementos que podem facilitar o processo de implementação do apoio contínuo na assistência ao parto.

\section{Métodos}

A elaboração da síntese de evidências seguiu a metodologia SUPPORT (Supporting Policy Relevant Reviews and Trials), que orienta a identificação de problemas relevantes a serem trabalhados, a realização dos processos de busca, extração e avaliação das evidências, bem como a aplicação desses achados na formulação das políticas de saúde ${ }^{19}$.

A busca de estudos foi realizada entre março e julho de 2018 nas seguintes bases de dados e sites de literatura científica: PubMed, Cochrane Library, Health Systems Evidence (HSE), Health Evidence (HE), Evidence for Informed Health Policymaking (PDQ-Evidence), Web of Science, Scopus, Embase, The International Network of Agencies for Health Technology Assessment (INAHTA), Center for Reviews and Dissemination (CRD), Canadian Agency for Drugs and Technologies in Health (CADTH) e Portal Regional da BVS. Para cada base foi desenvolvida uma estratégia de busca própria, com os termos "cesariana”, "cesárea”, "cesarean section", "abdominal delivery", "parto abdominal", "tocologia", "midwifery", "parteria”, "assistência ao parto”, "assistência tradicional ao nascimento", "obstetriz", "parteira”, "parturition", "birth", "vaginal birth", "childbirth", "humanization", "natural childbirth", "natural parturition". Foram utilizados filtros para obter revisões sistemáticas nos idiomas português, espanhol e inglês. Não houve restrição quanto à data de publicação. As buscas foram atualizadas em outubro de 2019 e não foram identificadas outras revisões sistemáticas para inclusão neste artigo.

Foram incluídas revisões com foco em intervenções não clínicas no contexto gestacional, cujos resultados estiveram relacionados à redução nas taxas de cesariana. A população de interesse era composta por gestantes classificadas como risco habitual e com gestações cefálicas e únicas. Intervenções não clínicas foram consideradas como aquelas aplicadas independentemente do encontro entre determinado profissional e usuário, no contexto do atendimento em saúde ${ }^{20}$.

Os processos de seleção de artigos, extração de dados e avaliação da qualidade metodológica de revisões sistemáticas foram realizados em dupli- cidade, e de forma independente, e as divergências foram resolvidas por consenso. A qualidade metodológica foi avaliada por meio da ferramenta Assessing the Methodological Quality of Systematic Reviews - AMSTAR, ${ }^{21}$ que contém 11 itens de avaliação, e as revisões sistemáticas incluídas foram classificadas como de qualidade baixa ( 0 a 3 itens avaliados positivamente), moderada ( 4 a 7) e alta ( 8 a 11).

\section{Resultados}

Nas buscas de literatura foram identificadas 6.493 revisões sistemáticas, conforme a Figura 1 . Após o processo de seleção, excluindo-se artigos repetidos e outros que não atendiam aos objetivos do trabalho, 17 revisões sistemáticas foram selecionadas e seus resultados permitiram elencar cinco opções para políticas de saúde: intervenções combinadas com foco em profissionais de saúde e na gestão dos serviços e sistemas de saúde; preparo da mulher para o parto normal durante o pré-natal; apoio contínuo às mulheres durante o trabalho de parto e parto; acesso a diferentes ambientes para o parto; e apoio ao parto vaginal após cesárea prévia. Para a opção de apoio contínuo, que será detalhada na sequência, foram incluídas quatro revisões sistemáticas, todas com metanálise.

De acordo com a avaliação da ferramenta AMSTAR e os critérios pré-estabelecidos de classificação, duas revisões foram consideradas de alta qualidade metodológica ${ }^{15,22}$, uma modera$\mathrm{da}^{23}$ e outra baixa ${ }^{24}$. O Quadro 1 traz a caracterização dos estudos incluídos.

O apoio contínuo foi oferecido por diferentes atores: doula, cônjuge/companheiro(a), profissional de saúde (obstetriz, enfermeira), membro da família, amigo(a) ou outra pessoa com alguma ou nenhuma formação especial em prestar apoio no trabalho de parto. Todos os estudos avaliaram o suporte oferecido em contexto hospitalar e nenhum deles encontrou danos relacionados à intervenção. As revisões foram publicadas no período de 1996 a 2017, e incluíram entre 1.252 e 15.858 participantes em 17 países (Austrália, África do Sul, Bélgica, Botsuana, Brasil, Canadá, Chile, Estados Unidos, Finlândia, França, Grécia, Guatemala, Irã, México, Nigéria, Tailândia e Turquia). A maioria dos estudos primários foram desenvolvidos em países de alta ou média renda e incluíram gestantes nulíparas.

Ao analisar os efeitos da intervenção em relação à cesariana (Quadro 2), o apoio contínuo 


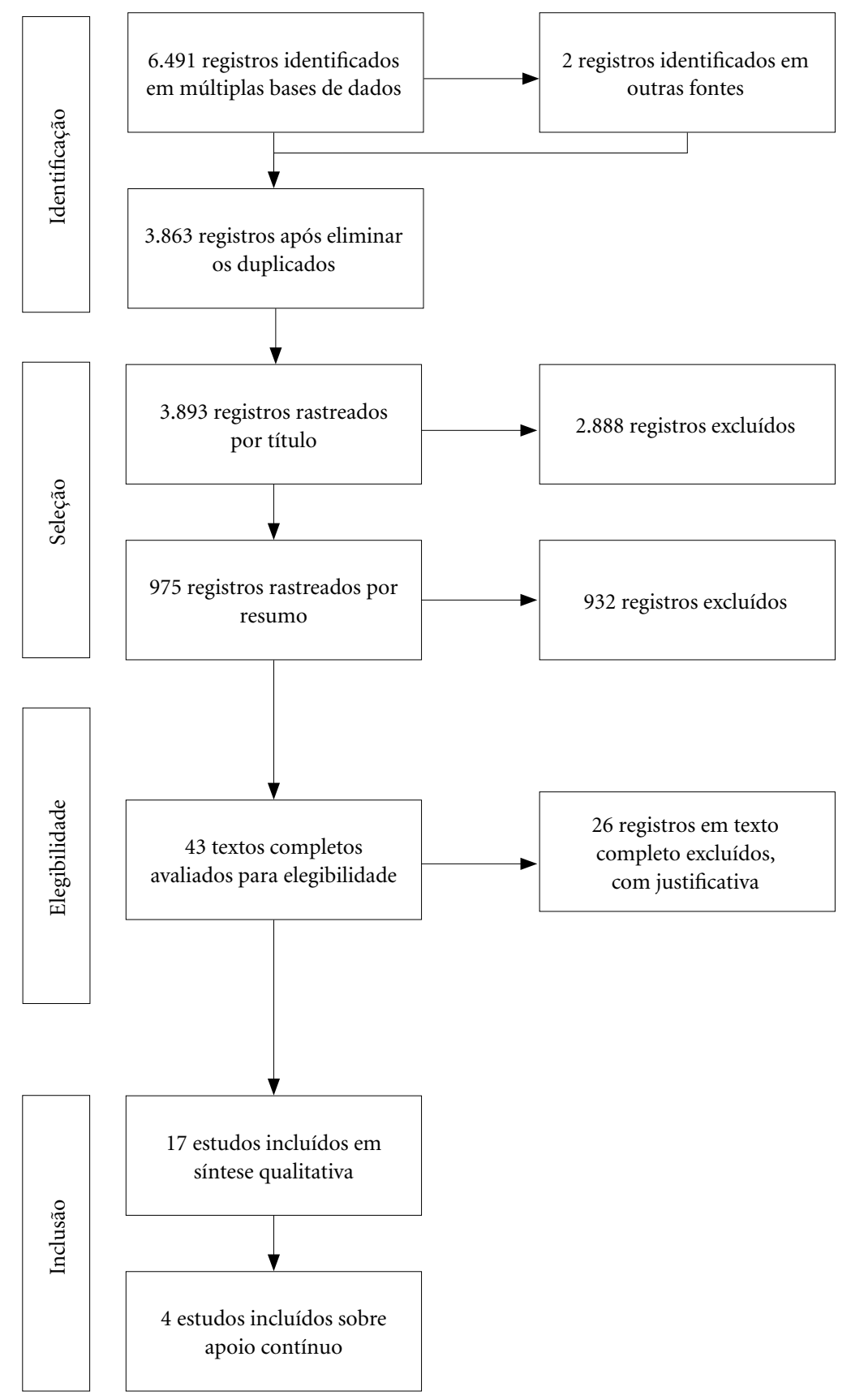

Figura 1. Fluxograma de seleção de estudos.

Fonte: Autores.

esteve associado a uma redução que variou entre $25 \%$ e $51 \%$. Uma das revisões comparou o apoio contínuo e intermitente prestado por uma pessoa na função de doula à ausência de apoio, e mostrou que o impacto na redução das taxas de cesariana e em outros desfechos (uso de fór- ceps, analgesia, ocitocina e duração do trabalho de parto) só foi significativo quando o apoio foi oferecido de forma contínua ${ }^{23}$.

Além das taxas de cesárea, o apoio contínuo reduziu as intervenções clínicas durante o trabalho de parto, como uso de ocitocina ${ }^{23,24}$, 
Quadro 1. Caracterização dos estudos incluídos.

\begin{tabular}{|c|c|c|}
\hline $\begin{array}{l}\text { Autor } \\
\text { (ano) }\end{array}$ & $\begin{array}{c}\text { Características } \\
\text { dos estudos } \\
\text { incluídos }\end{array}$ & Intervenção/ controle \\
\hline $\begin{array}{l}\text { Bohren, } \\
2017^{15}\end{array}$ & $\begin{array}{l}27 \text { ECR; } \\
\text { n = } 15.858 \\
\text { Países: } 16 \text { AR, } \\
9 \text { AMR; } 2 \\
\text { BMR }\end{array}$ & $\begin{array}{l}\text { Intervenção } \\
\text { Apoio contínuo - definição: qualquer combinação de medidas de conforto, apoio } \\
\text { emocional, fornecimento de informação e defesa às mulheres, providas pelo menos no } \\
\text { início do trabalho de parto (antes de } 6 \mathrm{~cm} \text { de dilatação) ou dentro da primeira hora } \\
\text { de admissão no hospital (para admissão de mulheres com } 6 \mathrm{~cm} \text { ou mais de dilatação), } \\
\text { até pelo menos o nascimento, sendo fornecido por uma pessoa cuja principal } \\
\text { responsabilidade era oferecer apoio às mulheres da forma mais continuamente } \\
\text { possível para o dado contexto } \\
\text { Responsável pelo apoio contínuo } \\
\text { Doula; companheiro ou outro acompanhante de escolha da mulher (alguns estudos } \\
\text { limitavam à pessoa do sexo feminino); obstetriz; alunos de obstetrícia; enfermeira; } \\
\text { educadora perinatal; amiga ou parente da mulher que recebia um treinamento sobre } \\
\text { trabalho de parto } \\
\text { Controle } \\
\text { Cuidado habitual (por exemplo, ausência de acompanhamento, cuidado intermitente } \\
\text { por enfermeiras ou obstetrizes) }\end{array}$ \\
\hline $\begin{array}{l}\text { Fortier, } \\
2015^{22}\end{array}$ & $\begin{array}{l}5 \text { ECR; } \\
\mathrm{n}=2.019 \\
\text { Países: } 4 \text { AR; } 1 \\
\text { AMR }\end{array}$ & $\begin{array}{l}\text { Intervenção } \\
\text { Apoio contínuo (sem definição) } \\
\text { Responsável pelo apoio contínuo } \\
\text { Doula } \\
\text { Controle } \\
\text { Cuidado obstétrico padrão }\end{array}$ \\
\hline $\begin{array}{l}\text { Scott, } \\
1999^{23}\end{array}$ & $\begin{array}{l}11 \text { ECR ( } 5 \\
\text { sobre apoio } \\
\text { contínuo } \\
\mathrm{n}=1.809 \text {, e } 6 \\
\text { sobre apoio } \\
\text { intermitente, } \\
\mathrm{n}=2.582) \\
\text { Países: } 8 \text { AR; } 1 \\
\text { AMR; } 2 \text { BMR }\end{array}$ & $\begin{array}{l}\text { Intervenção } \\
\text { Apoio contínuo ou intermitente - definição: apoio contínuo foi descrito como a } \\
\text { presença constante de alguém com a mulher ao longo do trabalho de parto e parto, } \\
\text { exceto para ir ao banheiro, logo após sua admissão no serviço ou entrada no estudo. } \\
\text { Definiu-se apoio intermitente quando a atendente do parto deixava a parturiente por } \\
\text { qualquer período de tempo que não apenas com o propósito de ir ao banheiro. Se nem } \\
\text { todas as mulheres do estudo tinham uma doula em tempo integral, com exceção da } \\
\text { saída para ir ao banheiro, esse estudo foi alocado no grupo de cuidado intermitente) } \\
\text { Responsável pelo apoio contínuo } \\
\text { Pessoas exercendo a função de doulas, com diferentes características (parteiras não } \\
\text { diplomadas - lay midwives), mulheres sem formação para atenção ao parto, obstetrizes } \\
\text { ou alunas de obstetrícia } \\
\text { Controle } \\
\text { Ausência de apoio. Em um dos estudos, o grupo controle era a presença de uma } \\
\text { acompanhante que não podia tocar a mulher ou se comunicar com ela }\end{array}$ \\
\hline $\begin{array}{l}\text { Zhang, } \\
1996^{24}\end{array}$ & $\begin{array}{l}5 \text { ECR. } \\
\mathrm{n}=1.252 \\
\text { Países: 2AR; } \\
\text { 1AMR; 2BMR }\end{array}$ & $\begin{array}{l}\text { Intervenção } \\
\text { Cuidado contínuo (presença de acompanhante a maior parte do tempo) } \\
\text { Responsável pelo apoio contínuo } \\
\text { Doula (4 ECR); doula em conjunto com o marido ou parceria (1 ECR) } \\
\text { Controle } \\
\text { Sem apoio (4 ECR); marido ou parceria (1 ECR) }\end{array}$ \\
\hline
\end{tabular}

ECR: ensaio clínico randomizado; AR: alta renda; AMR: alta média renda; BMR: baixa média renda. Classificação feita pelos autores desta revisão com base na categorização proposta pelo Banco Mundial, disponível em: https://ibraries.acm.org/binaries/content/assets/ libraries/archive/world-bank-list-of-economies.pdf.

Fonte: Autores.

analgesia ${ }^{15,23,24}$ e parto vaginal instrumental ${ }^{15,22-24}$. Também esteve correlacionado com menor duração do trabalho de parto ${ }^{15,23,24}$, menor probabilidade de recém-nascido com baixo escore de
Apgar aos cinco minutos ${ }^{15}$, início mais rápido da amamentação ${ }^{15}$, maior probabilidade de estar em amamentação exclusiva após seis semanas do parto $^{24}$, melhor recuperação da mulher durante o 
Quadro 2. Efeitos do apoio contínuo.

\begin{tabular}{|c|c|c|}
\hline $\begin{array}{l}\text { Autor } \\
\text { (Ano) }\end{array}$ & $\begin{array}{l}\text { Redução na taxa de } \\
\text { cirurgia cesariana }\end{array}$ & Melhoria em outros desfechos \\
\hline $\begin{array}{l}\text { Bohren, } \\
2017^{15}\end{array}$ & $\begin{array}{l}\text { Redução de } 25 \% \text { nas } \\
\text { taxas de cesariana } \\
(\mathrm{RR} 0,75 ; \mathrm{IC} 95 \% 0,64 \\
\left.\text { a } 0,88 ; \mathrm{I}^{2}=58 \%\right)\end{array}$ & $\begin{array}{l}\text { Maior probabilidade de parto vaginal espontâneo (RR 1,08; IC95\% 1,04 } \\
\text { a 1,12; } \mathrm{I}^{2}=61 \% ; 21 \text { ensaios, } 14.369 \text { mulheres); } \\
\text { Menor probabilidade de experiência negativa no parto (RR 0,69; IC 95\% } \\
0,59 \text { a } 0,79 ; \mathrm{I}^{2}=63 \% ; 11 \text { estudos, } 11.133 \text { mulheres); } \\
\text { Menor probabilidade de usar qualquer analgesia intraparto (RR 0,90; IC } \\
\text { 95\% 0,84 a 0,96; } \mathrm{I}^{2}=73 \% ; 15 \text { ensaios, } 12.433 \text { mulheres); } \\
\text { Menor tempo de trabalho de parto (Diferença de média -0,69 horas, } \\
\text { IC95\% -1,04 a -0,34; } \mathrm{I}^{2}=66 \% ; 13 \text { estudos, } 5.429 \text { mulheres); } \\
\text { Menor probabilidade de parto vaginal instrumental (RR 0,90; IC95\% } \\
0,85 \text { a } 0,96 ; \mathrm{I}^{2}=25 \% ; 19 \text { ensaios, } 14.118 \text { mulheres), } \\
\text { Menor probabilidade de analgesia regional (RR 0,93; IC95\% 0,88 a 0,99; } \\
\mathrm{I}^{2}=81 \% ; 9 \text { estudos, } 11.444 \text { mulheres), Menor probabilidade de recém- } \\
\text { nascido com Apgar baixo aos cinco minutos (RR 0,62; IC95\% 0,46 a 0,85; } \\
\mathrm{I}^{2}=17 \% ; 14 \text { ensaios, } 12.615 \text { mulheres); } \\
\text { Início mais rápido da amamentação ( } 1 \text { ensaio clínico, } 585 \text { mulheres (MD } \\
\text {-44,6 minutos, IC95\% -47,63 - 41,57) }\end{array}$ \\
\hline $\begin{array}{l}\text { Fortier, } \\
2015^{22}\end{array}$ & $\begin{array}{l}\text { Redução de } 42 \% \text { nas } \\
\text { taxas de cesariana } \\
\text { (OR } 0,68, \text { IC } 95 \% 0.47 \\
\text { a } 0.99, \mathrm{I}^{2}=58 \% \text { ) }\end{array}$ & $\begin{array}{l}\text { Redução da taxa de parto vaginal instrumental (OR 0,54, IC95\% 0,35 a } \\
0,92, \mathrm{I}^{2}=53 \% \text { ) }\end{array}$ \\
\hline $\begin{array}{l}\text { Scott, } \\
1999^{23}\end{array}$ & $\begin{array}{l}\text { Quando analisado } \\
\text { apenas o apoio } \\
\text { contínuo, a redução } \\
\text { de cesariana foi } \\
\text { de } 51 \%(\mathrm{OR}=0,49 \\
{[\mathrm{IC} 95 \% \text { : } 0.37 \text { a } 0.65 ;} \\
\text { p }<0.05])\end{array}$ & $\begin{array}{l}\text { A análise dos estudos de apoio intermitente de doulas não mostrou } \\
\text { diferença estatisticamente significativa para os cinco desfechos } \\
\text { analisados. Entretanto, a análise dos estudos sobre apoio contínuo } \\
\text { dessa profissional, em comparação com a ausência de apoio, reduziu a } \\
\text { probabilidade de uso de analgesia, condução do parto com ocitocina e } \\
\text { fórceps em, respectivamente } 36 \% \text { (OR=0,64 [IC95\% 0,49-0,85]), } 71 \% \\
(\mathrm{OR}=0,29 \text { [ IC95\% 0,20-0,40]) e } 57 \% \text { (OR=0,43 [IC95\% 0,28-0,65). } \\
\text { Mulheres em apoio contínuo tiveram redução na duração do trabalho de } \\
\text { parto em comparação com os controles }\end{array}$ \\
\hline $\begin{array}{l}\text { Zhang, } \\
1996^{24}\end{array}$ & $\begin{array}{l}\text { Redução de } 46 \% \text { nas } \\
\text { taxas de cesariana } \\
\text { (RR 0,54; IC } 95 \% 0,4 \\
\text { a } 0,7) \\
\text { No estudo em síntese } \\
\text { narrativa, a taxa de } \\
\text { cesárea foi similar em } \\
\text { ambos os grupos }\end{array}$ & $\begin{array}{l}\text { Redução de 2,8 horas no trabalho de parto (IC95\% 2,2-3,4); } \\
\text { Menor frequência de uso de ocitocina (RR 0,44; IC95\% 0,3-0,6); } \\
\text { Maior probabilidade de parto vaginal espontâneo (RR 2,01, IC95\% } \\
1,5-2,7) \text {; } \\
\text { Menor chance de uso de fórceps (redução de 54\%; RR 0,46; IC95\% } \\
0,3-0,7 \text { ); } \\
\text { Redução no uso de analgesia ( } 20 \% \text { menor no grupo não incluído na } \\
\text { metanálise, entretanto nenhuma diferença substancial foi observada no } \\
\text { uso de analgesia nos demais estudos); } \\
\text { Resultados também mostraram menos episódios de febre nas mulheres } \\
\text { (1 estudo); menos recém-nascidos em internação por mais de } 48 \text { horas, } \\
\text { transferência para UTI e redução nos episódios de sepse neonatal (1 } \\
\text { estudo); Mulheres com apoio contínuo também permaneceram mais } \\
\text { ativas na primeira hora após o parto, acariciaram mais o recém-nascido } \\
\text { e sorriram mais para eles ( } 1 \text { estudo); As puérperas em apoio contínuo } \\
\text { também relataram menor ansiedade no trabalho de parto e parto, menor } \\
\text { intensidade da dor no parto e pós-parto e melhor vivência do processo. } \\
\text { Após } 6 \text { semanas, as mulheres que receberam apoio contínuo também } \\
\text { estavam mais propensas a estarem em amamentação exclusiva (1 estudo) }\end{array}$ \\
\hline
\end{tabular}

Fonte: Autores.

puerpério $^{24}$, aumento da satisfação com a experiência do nascimento ${ }^{24}$ e maiores chances de parto vaginal espontâneo ${ }^{15,24}$.
O apoio contínuo foi efetivo para mulheres de diferentes contextos sociodemográficos e econômicos ${ }^{15,22}$. Contudo, análise de subgrupo 
realizada por Bohren e colaboradores mostrou que a taxa de cesárea foi reduzida de forma mais expressiva em países de média renda, em relação a regiões mais ricas ${ }^{15}$. Além disso, a redução foi mais expressiva quando uma pessoa na função de doula exerceu o apoio contínuo, quando o acompanhante era de escolha da mulher e em contextos em que a epidural não estava livremente disponível ${ }^{15}$. Os benefícios do apoio contínuo também foram econômicos, observados a partir da redução dos custos hospitalares ${ }^{23}$.

\section{Discussão}

Desde a década de 1990, com o apoio da popularização da saúde baseada em evidências, o movimento de mulheres vem demonstrando a importância do apoio contínuo no processo de nascimento ${ }^{25}$. As revisões sistemáticas incluídas nesta síntese de evidências reforçam a importância desse apoio. Os resultados destacam seu efeito benéfico na redução das cirurgias cesarianas, das chances de recém-nascido com baixo escore de Apgar, no uso de intervenções clínicas no trabalho de parto, na duração do trabalho de parto, nas melhorias na amamentação e na recuperação da mulher durante o puerpério e no aumento da satisfação com a experiência do nascimento e das chances de parto vaginal espontâneo.

Esses benefícios podem ser explicados pela maior segurança e confiança propiciadas às mulheres, facilitando a fisiologia do parto e reduzindo a frequência de intervenções clínicas ${ }^{15}$. Além disso, com acompanhamento constante, a parturiente tende a se movimentar mais e a adotar diferentes posições, o que facilita o trabalho de parto. Essas mulheres tendem também a receber uma oferta maior de métodos não farmacológicos para alívio da dor e a se sentirem mais seguras, especialmente onde os(as) profissionais estão muito sobrecarregados(as) para perceber intercorrências clínicas com facilidade ${ }^{15}$.

A análise dos dados da pesquisa "Nascer no Brasil" também indicou que a presença de acompanhante no trabalho de parto esteve relacionada à maior adoção de métodos não farmacológicos para alívio da dor e redução no uso de enema e tricotomia. O apoio no momento do parto também propiciou maior adoção de diferentes posições para o nascimento, permanência em leito PPP (pré-parto, parto, pós-parto), contato pele a pele e redução no uso da manobra de Kristeller. Por outro lado, não houve impacto no uso de ocitocina e de episiotomia, e essas mulheres rece- beram mais amniotomia e analgesia ${ }^{26}$. De modo similar, uma das revisões incluídas neste estudo também não encontrou correlação entre o apoio contínuo e a redução no uso de analgesia ${ }^{22}$, e duas não mostraram redução significativa no uso de ocitocina no trabalho de parto ${ }^{15,22}$.

Os resultados da síntese de evidências revelaram também que parcela significativa das mulheres incluídas nos estudos eram nulíparas. Portanto, ao prevenir a primeira cesárea, o apoio contínuo ainda tem potencial de reduzir as chances de cesáreas futuras. Isso ocorre porque muitos profissionais ainda indicam a realização dessa cirurgia para mulheres com cesárea anterior ${ }^{15}$, a despeito das evidências que comprovam que o parto vaginal após cesárea prévia (VBAC) é uma opção segura para a maioria da população ${ }^{27}$.

Com a presença do apoio, as experiências negativas no parto foram reduzidas. Além de assegurar respeito a suas crenças e valores socioculturais, as mulheres consideraram que suas expectativas foram superadas ${ }^{15}$. Estudos qualitativos realizados no contexto brasileiro corroboram esses resultados e mostram que a participação ativa do acompanhante é capaz de trazer conforto físico e emocional às mulheres, redução do sentimento de solidão e sensação de maior segurança e tranquilidade ${ }^{28,29}$. Da mesma forma, o apoio contínuo pode facilitar a comunicação entre a mulher e a equipe de saúde, bem como a expressão dos valores e desejos da parturiente em situações nas quais ela esteja, ou se sinta, impossibilita$\mathrm{da}^{30}$. As doulas, por exemplo, exercem um papel importante ao fornecer informações técnicas às mulheres durante a gestação, parto e pós-parto, possibilitando que o processo de nascimento seja melhor vivenciado e compreendido como um evento fisiológico. Nesse sentido, as mulheres tendem a se sentir menos ansiosas e mais seguras para tirar dúvidas e dialogar com os profissionais de saúde ${ }^{30}$.

Os achados da síntese de evidências mostraram benefícios de ordem econômica ${ }^{23}$, o que também foi indicado em estudo de custo-efetividade realizado no contexto dos Estados Unidos. A presença da doula durante o trabalho de parto mostrou potencial de redução de custos globais dos nascimentos, gerando economia para os serviços por meio de uma diminuição importante das taxas de cesárea, da prematuridade neonatal e, consequentemente, dos custos relacionados aos cuidados neonatais especializados, a hospitalizações frequentes e à assistência médica de longo prazo ${ }^{31}$.

Garantir apoio contínuo às mulheres, em especial nos países de baixa e média renda, pode ser 
uma ferramenta oportuna para o empoderamento da parturiente, favorecendo um cuidado culturalmente sensível e estimulando a proteção dos seus direitos, o que beneficia a equidade em saú$\mathrm{de}^{30}$. No contexto da assistência obstétrica brasileira, por exemplo, no qual uma em cada quatro mulheres relata episódios de violência obstétri$\mathrm{ca}^{32}$, o apoio contínuo pode ajudar no combate a essa forma de violência de gênero. Em especial, seriam favorecidas mulheres negras, pois estão mais propensas a sofrer essa violência e a receber um cuidado de menor qualidade devido ao racismo institucional. Mulheres em situação de rua, privadas de liberdade, imigrantes ou com alguma vulnerabilidade social e econômica podem se beneficiar ainda mais do apoio contínuo ${ }^{14}$.

Entretanto, apesar do aumento importante na proporção de mulheres que contam com a presença de acompanhante no trabalho de parto e nascimento nos últimos anos, nem sempre esse direito é respeitado no Brasili ${ }^{8}$. Essa também é uma realidade em outros países da América Latina e do Caribe, onde são incipientes as regulamentações que permitem a presença de acompanhante no contexto do nascimento; ou, quando existentes, não são conhecidas pelos profissionais, gestores e usuários dos serviços ${ }^{33}$. Entre as barreiras à implementação da presença do acompanhante no parto, observa-se a falta de incentivo dos gestores e de recursos humanos e financeiros para adaptação das alas obstétricas, treinamento dos profissionais e contratação de trabalhadores com essa finalidade ${ }^{34}$. Portanto, para favorecer a ampliação da oferta desse cuidado às mulheres e recém-nascidos no país, alguns aspectos relacionados à implementação dessa prática são pontuados e discutidos na sequência, com foco no que merece particular consideração pelos gestores e profissionais de saúde.

Primeiramente, é necessário garantir que nenhuma mulher fique sozinha ao longo do processo de nascimento e puerpério, a não ser por curtos intervalos de tempo ou nos casos em que ela solicite mais privacidade ${ }^{35}$. A OMS preconiza, e o Estado brasileiro determinou em lei, que todas as gestantes têm direito a acompanhante de sua livre escolha durante o período de trabalho de parto, parto e pós-parto, tanto no SUS quanto em instituições a ele conveniadas ${ }^{36,37}$. A Rede Cegonha e as Diretrizes Nacionais de Assistência ao Parto Normal reiteram essa importância ${ }^{38,39}$.

A lei é válida para parto normal ou cesariana e a presença do(a) acompanhante (inclusive se for adolescente) não pode ser impedida pelo hospital ou por qualquer membro da equipe de saúde, sob qualquer exigência. Na saúde suplementar, a Agência Nacional de Vigilância Sanitária estabelece que os serviços devem permitir a presença de acompanhante de livre escolha da mulher no acolhimento, trabalho de parto, parto e pós-parto imediato ${ }^{40}$. Além disso, a ANS determina que os planos de saúde com cobertura de obstetrícia arquem com todas as despesas do acompanhante durante este período ${ }^{41}$. Entretanto, em alguns locais as mulheres precisam escolher entre $o(a)$ acompanhante e a doula sob justificativas diversas, como a falta de espaço para circulação da equipe. Entretanto, é preciso destacar que a presença da doula não invalida a importância da participação de um(a) acompanhante no processo de nascimento e vice-versa ${ }^{42}$.

Portanto, são necessárias adequações relativas às práticas dos trabalhadores nos serviços de atenção ao parto para que se possa realizar o apoio contínuo "um para um" por doulas, obstetrizes e enfermeiras obstetras. Inclusive, a capacitação apropriada é bem-vinda, considerando que profissionais de saúde muitas vezes carecem das habilidades requeridas para apoio ao trabalho de parto $^{15}$. O apoio contínuo oferecido por profissionais da assistência obstétrica nos serviços de saúde depende de condições adequadas de trabalho, que envolvem a eliminação de práticas de desvio de função e maior número de trabalhadores(as) por período. Destaca-se que os(as) profissionais da área da obstetrícia precisam manter seus postos de trabalho vinculados à obstetrícia propriamente ${ }^{43}$.

Para tratar dessa questão de forma específica, com base em um diálogo deliberativo realizado com a participação de diversos atores sociais interessados nessa temática, sugere-se a criação e implantação de uma matriz de competências de cada atuação dentro dos serviços, pactuando, entre gestão e trabalhadores(as), as funções a serem desempenhadas por cada um(a), respeitando habilidades, a autonomia profissional e valorizando os(as) trabalhadores(as) do serviço ${ }^{14}$. Conforme apontado em uma das revisões sistemáticas incluídas, apesar de o apoio contínuo oferecido por profissionais do serviço ser importante e positivo para mulheres e recém-nascidos, ter alguém desempenhando a função de doula também é relevante, uma vez que nem sempre os profissionais da obstetrícia conseguem oferecer suporte suficiente às mulheres, pois têm de realizar outras tarefas durante a assistência ${ }^{22}$.

Outra barreira importante para permanência de acompanhantes, sobretudo do sexo masculino, refere-se às limitações de espaço físico nas 
unidades obstétricas. Em enfermarias compartilhadas, a presença do acompanhante pode ser vista como um fator de violação da privacidade das demais mulheres alocadas no mesmo espaço. Por isso, as instituições de saúde devem estar preparadas para receber esse acompanhante, bem como proporcionar ou acolher o profissional que fornece o apoio contínuo ${ }^{37}$.

Para facilitar a presença de acompanhantes e a atuação de doulas no SUS, diversas medidas passaram a ser adotadas. A estruturação da Rede Cegonha, por exemplo, coordenada pelo Ministério da Saúde, determinou diretrizes e normas de ambiência para adequação e habilitação dos locais de parto e nascimento. As orientações se baseiam na necessidade de espaço físico para que se cumpra o direito ao acompanhante e se caminhe para a superação do modelo biologicista e medicalizante de atenção ao parto ${ }^{44}$.

Conforme já discutido, a análise dos primeiros resultados da Rede Cegonha e do Projeto Parto Adequado referentes ao ano de 2017 mostraram um avanço na presença de acompanhantes durante o parto em comparação com os dados da pesquisa "Nascer no Brasil", conduzida entre fevereiro de 2011 e outubro de 2012. O aumento foi de $164 \%$ nas maternidades públicas (de $31,8 \%$ para $83,9 \%$ ) e $73 \%$ (de $55,8 \%$ para $96,8 \%$ ) no serviço privado ${ }^{8}$. Os dados revelam que políticas públicas bem desenhadas e o comprometimento das gestões federal e local com a transformação do modelo podem mudar a realidade da assistência ao parto no país. Por isso precisam ser mantidas, incentivadas e expandidas, garantindo que todas as mulheres tenham seus direitos garantidos.

No Brasil, desde 2013 as competências e limitações profissionais das doulas estão descritas no rol da Classificação Brasileira de Ocupações, facilitando a criação de políticas públicas que incluam a atuação da categoria nos estabelecimentos de saúde. Leis que regulamentam a atuação dessas profissionais e obrigam estabelecimentos públicos e privados a garantirem a presença de doula para a parturiente durante todo o período de trabalho de parto, parto e puerpério já estão instituídas em 16 estados, 12 capitais, e 67 municípios, além de nove projetos de lei em andamento em diferentes estados e municípios (Carta da ADOULA/RJ à CSSF, disponível mediante solicitação às autoras).

Em diversas instituições do país existem programas voltados à atuação de doulas voluntárias. Por exemplo, o que foi iniciado em 1997 no Hospital Sofia Feldman, em Belo Horizonte ${ }^{45}$, e mais recentemente o Hospital da Mulher Dra. Nise da Silveira, em Maceió, que se tornou o primeiro serviço a contratar doulas para atenção às parturientes pelo SUS ${ }^{46}$. No município de São Paulo, com o objetivo de assegurar o cumprimento da demanda na rede pública, a Secretaria Municipal de Saúde instituiu o Programa de Doulas Voluntárias em 2017, permitindo o acompanhamento de gestantes, parturientes e puérperas nas maternidades, hospitais e demais equipamentos da rede municipal de saúde ${ }^{47}$.

Em âmbito nacional, encontra-se em tramitação na Câmara dos Deputados texto que dispõe sobre o exercício profissional da atividade de doula ${ }^{48}$. Ao ser aprovado, o projeto pretende regulamentar o exercício da doulagem em todo o território nacional, assegurando sua prática em estabelecimentos de saúde públicos e privados, casas de parto e domicílio, sendo a assistência dela de livre escolha da mulher e independente da presença de um(a) acompanhante.

Para atender às demandas de mulheres migrantes latino-americanas, existe o projeto de saúde da mulher migrante latino-americana em São Paulo, promovido pela ONG Equipe de Base Warmis Convergência das Culturas. São voluntárias que atuam na transformação da realidade e na melhoria das condições de vida desse grupo, por meio de atividades diversas no município de São Paulo. O projeto consiste em proporcionar ajuda às imigrantes, acolhendo-as, auxiliando-as no acesso aos serviços de saúde, protegendo seus direitos, informando e dando apoio, especialmente no período gravídico e puerperal ${ }^{49}$.

As ações descritas são importantes para a promoção do apoio contínuo e a criação de um ambiente seguro para o parto e nascimento. São atividades que permitem o acolhimento das demandas psicossociais da mulher, a oferta de pessoal técnico capacitado e humanizado e a participação informada das mulheres nos processos de decisão, inclusive quando houver necessidade de alguma intervenção clínica ${ }^{37}$. Entre as lacunas de pesquisa identificadas, as revisões sistemáticas recomendam: investigar o impacto dos distintos modelos de atenção obstétrica nos efeitos do apoio contínuo; conduzir mais trabalhos em países de baixa e média renda; avaliar o efeito do apoio contínuo na depressão pós-parto, amamentação e prevenção da violência obstétrica; e realizar avaliações econômicas.

Além disso, com o surgimento e expansão da covid-19 desde novembro de 2019, o impacto da pandemia na mudança dos protocolos de atenção ao parto passa a merecer destaque, em parti- 
cular no que diz respeito à garantia dos direitos das mulheres, como o acesso ao apoio contínuo nos serviços. Tendo em vista as mudanças fisiológicas no corpo da mulher durante a gestação e pós-parto e o pequeno número de estudos até o momento que avaliem os efeitos do SARS-CoV-2 nos desfechos gestacionais ${ }^{50}$, mudanças são necessárias para proteger as mulheres, os recémnascidos e também os profissionais envolvidos no cuidado do binômio mãe-bebê. Entretanto, não há justificativas para que as mulheres, diagnosticadas ou não com covid-19, tenham o seu direito ao acompanhante negado, tampouco que doulas sejam proibidas de prestar assistência, o que vem acontecendo no Brasil e no mundo ${ }^{18,51,52}$. Em alguns contextos, essas limitações à presença de acompanhante estão sendo flexibilizadas, mas ainda há protocolos que não recomendam a presença de doulas nos serviços ${ }^{53}$. O impacto dessas restrições precisa ser estudado, pois há evidências de que o próprio cenário da pandemia tem despertado sensações de medo, solidão e angústia nas mulheres durante o parto. Logo, limitar uma fonte de apoio às parturientes talvez seja outro fator negativo a ser acumulado na experiência de nascimento $0^{54}$.

Por fim, cabe destacar que características das populações incluídas nas análises são uma limitação desta síntese de evidências. A maior parte dos resultados foi obtida em países de alta renda, principalmente devido à desigualdade na disseminação de pesquisas de países mais pobres, limitando a extrapolação dos resultados para esses contextos. Outra limitação se refere ao baixo número de revisões sistemáticas incluídas. Apesar disso, elas abarcam um contingente amplo de estudos primários e participantes em suas comparações por metanálise, incluídos a partir de busca abrangente na literatura e método sistemático de seleção, o que também permite sua validação e reprodução por fontes externas. A falta de detalhes sobre os atores que ofereceram o apoio contínuo e os contextos e modelos obstétricos no qual a intervenção foi investigada também limita a extrapolação dos resultados, por isso é importante que estudos futuros detalhem melhor esses aspectos.

\section{Considerações finais}

O parto é um evento histórico e socialmente construído. Esse momento da vida também pode refletir as iniquidades de gênero, ainda tão estruturadas nas sociedades, pois frequentemente são relatados episódios de abusos e violências. Nesse sentido, os estudos apresentados e analisados nesta revisão explicitam diversos benefícios que o apoio contínuo no parto traz à mulher e ao recém-nascido, inclusive para além de garantir seus direitos e sua segurança, uma vez que favorecem a fisiologia do trabalho de parto e o cumprimento dos princípios da humanização e do cuidado integral em saúde.

As evidências na literatura científica sugerem que o apoio contínuo foi efetivo para a redução das taxas da cirurgia cesariana, principalmente quando o suporte foi oferecido por uma pessoa na função de doula, o que reforça o estímulo à participação dessas profissionais nos serviços de saúde. Da mesma forma, a participação de outros atores também resultou na redução das taxas de cesariana. Entre eles, os profissionais que prestam assistência obstétrica, em especial no modelo de cuidado um para um; o acompanhante de escolha da mulher; ou mesmo outras pessoas designadas para apoiar as gestantes no parto. Independentemente, todos esses devem ter sua entrada garantida nos serviços. Ressalta-se, ainda, que as atividades das profissionais da doulagem não podem ser substituídas por outras figuras que compõem o ambiente de parto e nascimento, da mesma forma que essa não substitui a presença de um(a) familiar ou profissional da área técnica.

O apoio contínuo deve ser considerado essencial para a garantia de um cuidado respeitoso, seguro e adequado durante o trabalho de parto e parto. Por meio dele, protege-se a dignidade, privacidade e confidencialidade da mulher, possibilitando uma escolha livre e informada nesse momento. A efetivação do apoio contínuo tem potencial para reduzir os custos hospitalares, além de ser considerada um marco importante para segurança, qualidade do cuidado ofertado e respeito aos direitos das mulheres. Dessa forma, entende-se que a estratégia é importante para a adoção e manutenção nos serviços de saúde.

Em meio ao cenário atual de crise na saúde mundial causada pelo SARS-CoV-2, gestores, serviços e profissionais de saúde devem estar cada vez mais vigilantes à manutenção dos direitos das mulheres, como ao assegurar o apoio contínuo durante o trabalho de parto e parto. Assim, mantém-se o foco nos avanços que são almejados para a atenção ao parto e ao nascimento na atualidade e no mundo pós-pandemia. 


\section{Colaboradores}

CF Oliveira participou da análise e interpretação dos resultados e redigiu a versão inicial do manuscrito. TS Toma, MC Bortoli e C Setti foram responsáveis pela concepção e desenho do estudo e analisaram e interpretaram os resultados. CD Luquine Júnior contribuiu na interpretação dos resultados. Todos os autores participaram da escrita e revisão crítica do texto, tendo aprovado a versão final a ser publicada.

\section{Financiamento}

O projeto inicial de pesquisa foi contemplado na "Chamada pública de apoio a projetos de tradução do conhecimento no âmbito da rede de políticas informadas por evidências (EVIPNet Brasil) para o fortalecimento do Sistema Único de Saúde (SUS)", publicada em 2017 e financiada com recursos oriundos de Termo de Cooperação no 91 entre Ministério da Saúde e Organização Pan-Americana da Saúde.

\section{Referências}

1. Sandall J, Tribe RM, Avery L, Mola G, Visser GH, Homer CS, Gibbons D, Kelly NM, Kennedy HP, Kidanto H, Taylor P, Temmerman M. Short-term and long-term effects of caesarean section on the health of women and children. Lancet 2018; 392(10155):13491357.

2. Chen C, Yan Y, Gao X, Xiang S, He Q, Zeng G, Liu $\mathrm{S}$, Sha $\mathrm{T}$, Li L. Influences of Cesarean Delivery on Breastfeeding Practices and Duration: A Prospective Cohort Study. J Hum Lact 2018; 34(3):526-534.

3. World Health Organization (WHO), Human Reproductive Programme (HRP). WHO statement on caesarean section rates [Internet]. Geneva: WHO; 2015. [acessado 2020 Mai 10]. Disponível em: https://www. who.int/reproductivehealth/publications/maternal_ perinatal_health/cs-statement/en/

4. Ye J, Betrán AP, Guerrero Vela M, Souza JP, Zhang J. Searching for the optimal rate of medically necessary cesarean delivery. Birth 2014; 41(3):237-244.

5. The Lancet. Stemming the global caesarean section epidemic. Lancet 2018; 392(10155):1279.

6. Boerma T, Ronsmans C, Melesse DY, Barros AJD, Barros FC, Juan L, Moller AB, Say L, Hosseinpoor AR, Yi M, Rabello Neto DL, Temmerman M. Global epidemiology of use of and disparities in caesarean sections. Lancet 2018; 392(10155):1341-1348.

7. Leal MC, Silva AAM, Dias MAB, Gama SGN, Rattner D, Moreira ME, Theme Filha MM, Domingues RMSM, Pereira APE, Torres JA, Bittencourt SDA, D’orsi E, Cunha AJ, Leite AJM, Cavalcante RS, Lansky S, Diniz CSG, Szwarcwald CL. Birth in Brazil: national survey into labour and birth. Reprod Health 2012; 9(1):15.

8. Leal MC, Bittencourt SA, Esteves-Pereira AP, Ayres BVS, Silva LBRAA, Thomaz EBAF, Lamy ZC, Nakamura-Pereira M, Torres JA, Gama SGN, Domingues RMSM, Vilela MEA. Avanços na assistência ao parto no Brasil: resultados preliminares de dois estudos avaliativos. Cad Saude Publica 2019; 35(7):e00223018.

9. Patah LEM, Malik AM. Models of childbirth care and cesarean rates in different countries. Rev Saude Publica 2011; 45(1):185-194.

10. Victora CG, Aquino EM, Leal MC, Monteiro CA, Barros FC, Szwarcwald CL. Maternal and child health in Brazil: progress and challenges. Lancet 2011; 377(9780):1863-1876.

11. Diniz CSG, Chacham AS. O “corte por cima” e o "corte por baixo": o abuso de cesáreas e episiotomias em São Paulo. Questões de Saúde Reprodutiva 2006; 1(1):80-91.

12. Faúndes A, Cecatti JG. A operação cesárea no Brasil: incidência, tendências, causas, conseqüências e propostas de ação. Cad Saude Publica 1991; 7(2):150-173.

13. Rattner D, Moura EC. Nascimentos no Brasil: associação do tipo de parto com variáveis temporais e sociodemográficas. Rev Bras Saude Mater Infant 2016; 16(1):39-47.

14. Brasil. Ministério da Saúde (MS). Secretaria de Ciência, Tecnologia e Insumos Estratégicos. Departamento de Ciência e Tecnologia. Síntese de evidências para políticas de saúde: reduzindo as taxas de cirurgia cesariana no Brasil. Brasília: MS; no prelo. 
15. Bohren MA, Hofmeyr GJ, Sakala C, Fukuzawa RK, Cuthbert A. Continuous support for women during childbirth. Cochrane Database Syst Rev 2017; 7:CD003766.

16. Diniz CSG. Humanização da assistência ao parto no Brasil: os muitos sentidos de um movimento. Cien Saude Colet 2005; 10(3):627-637.

17. Leal MC, Bittencourt SA, Esteves-Pereira AP, Ayres BVS, Silva LBRAA, Thomaz EBAF, Lamy ZC, Nakamura-Pereira M, Torres JA, Gama SGN, Domingues RMSM, Vilela, MEA. Avanços na assistência ao parto no Brasil: resultados preliminares de dois estudos avaliativos. Cad Saude Publica 2019; 35(7):e00223018.

18. Davis-Floyd R, Gutschow K, Schwartz DA. Pregnancy, Birth and the covid-19 Pandemic in the United States. Med Anthropol 2020; 39(5):413-427.

19. Lavis JN, Oxman AD, Lewin S, Fretheim A. SUPPORT Tools for evidence-informed health Policymaking (STP). Heal Res Policy Syst 2009; 7(Suppl. 1):I1.

20. World Health Organization (WHO). WHO recommendations non-clinical interventions to reduce unnecessary caesarean sections [Internet]. Geneva: WHO; 2018. [acessado 2020 Mai 15]. Disponível em: https:// www.who.int/reproductivehealth/publications/nonclinical-interventions-to-reduce-cs/en/

21. Shea BJ, Grimshaw JM, Wells GA, Boers M, Andersson N, Hamel C, Porter AC, Tugwell P, Moher D, Bouter LM. Development of AMSTAR: a measurement tool to assess the methodological quality of systematic reviews. BMC Med Res Methodol 2007; 7(1):10.

22. Fortier JH, Godwin M. Doula support compared with standard care: meta-analysis of the effects on the rate of medical interventions during labour for low-risk women delivering at term. Can Fam Physician 2015; 61(6):e284-e292.

23. Scott KD, Berkowitz G, Klaus M. A comparison of intermittent and continuous support during labor: A meta-analysis. Am J Obstet Gynecol 1999; 180(5):10541059.

24. Zhang J, Bernasko JW, Leybovich E, Fahs M, Hatch MC. Continuous labor support from labor attendant for primiparous women: a meta-analysis. Obstet $G y$ necol 1996; 88(4):739-744.

25. Diniz CSG, D’Orsi E, Domingues RMSM, Torres JA, Dias MAB, Schneck CA, Lansky S, Teixeira NZF, Rance S, Sandall J. Implementation of the presence of companions during hospital admission for childbirth: data from the Birth in Brazil national survey. Cad Saude Publica 2014; 30(Supl. 1):S1-S14.

26. Monguilhott JJC, Brüggemann OM, Freitas PF, D’Orsi E. Nascer no Brasil: a presença do acompanhante favorece a aplicação das boas práticas na atenção ao parto na região Sul. Rev Saude Publica 2018; 52:1-11.

27. Guise J-M, Denman MA, Emeis C, Marshall N, Walker M, Fu R, Janik R, Nygren P, Eden KB, McDonagh M. Vaginal Birth After Cesarean: new insights on maternal and neonatal outcomes. Obstet Gynecol 2010; 115(6):1267-1278.

28. Motta CCL, Crepaldi MA. O pai no parto e apoio emocional: a perspectiva da parturiente. Paidéia (Ribeirão Preto) 2005; 15(30):105-118.
29. Dodou HD, Rodrigues DP, Guerreiro EM, Guedes MVC, Lago PN, Mesquita NS. The contribution of the companion to the humanization of delivery and birth: perceptions of puerperal women. Esc Anna Nery 2014; 18(2):262-269.

30. Bohren MA, Berger BO, Munthe-Kaas H, Tunçalp Ö. Perceptions and experiences of labour companionship: a qualitative evidence synthesis. Cochrane Database Syst Rev 2019; 3:CD012449.

31. Kozhimannil KB, Hardeman RR, Alarid-Escudero F, Vogelsang CA, Blauer-Peterson C, Howell EA. Modeling the cost-effectiveness of doula care associated with reductions in preterm birth and cesarean delivery. Birth 2016; 43(1):20-27.

32. Fundação Perseu Abramo. Mulheres brasileiras e gênero nos espaços público e privado [Internet]. São Paulo: Fundação Perseu Abramo; 2010. [acessado 2020 Mai 10]. Disponível em: https://fpabramo.org.br/publicacoes/wp-content/uploads/sites/5/2017/05/pesquisaintegra_0.pdf

33. De Mucio B, Binfa L, Ortiz J, Portela A. Status of national policy on companion of choice at birth in Latin America and the Caribbean: gaps and challenges. Rev Panam Salud Pública 2020; 44:e19.

34. Kabakian-Khasholian T, Portela A. Companion of choice at birth: factors affecting implementation. BMC Pregnancy Childbirth 2017; 17(1):265.

35. Brasil. Ministério da Saúde (MS). Secretaria de Ciência, Tecnologia e Insumos Estratégicos. Departamento de Gestão e Incorporação de Tecnologias em Saúde. Diretrizes nacionais de assistência ao parto normal: versão resumida. Brasília: MS; 2017.

36. Brasil. Lei $\mathrm{n}^{\circ} 11.108$, de 7 de abril de 2005. Altera a Lei no 8.080, de 19 de setembro de 1990, para garantir às parturientes o direito à presença de acompanhante durante o trabalho de parto, parto e pós-parto imediato, no âmbito do Sistema Único de Saúde (SUS). Brasília: Diário Oficial da União 2005; 8 abr.

37. World Health Organization (WHO). WHO recommendations intrapartum care for a positive childbirth experience [Internet]. Geneva: WHO; 2018. [acessado 2020 Mai 15]. Disponível em: https://www.who.int/ reproductivehealth/publications/intrapartum-careguidelines/en/

38. Brasil. Ministério da Saúde (MS). Gabinete do Ministro. Portaria $n^{\circ} 2.351$, de 5 de outubro de 2011. Altera a Portaria no 1.459/GM/MS, de 24 de junho de 2011, que institui, no âmbito do Sistema Único de Saúde (SUS), a Rede Cegonha. Brasília: Diário Oficial da União 2011; 6 out.

39. Brasil. Ministério da Saúde (MS). Diretrizes nacionais de assistência ao parto normal: versão resumida [Internet]. Brasília: MS; 2017. [acessado 2020 Jun 2]. Disponível em: https://pesquisa.bvsalud.org/bvsms/ resource/pt/mis-39026

40. Brasil. Ministério da Saúde (MS). Agência Nacional de Vigilância Sanitária. Resolução no 36 , de 3 de junho de 2008. Dispõe sobre Regulamento Técnico para Funcionamento dos Serviços de Atenção Obstétrica e Neonatal. Brasília: Diário Oficial da União 2008; 4 jun. 
41. Brasil. Ministério da Saúde (MS). Agência Nacional de Saúde Suplementar. Resolução Normativa no 428, de 7 de novembro de 2017. Atualiza o Rol de Procedimentos e Eventos em Saúde, que constitui a referência básica para cobertura assistencial mínima nos planos privados de assistência à saúde, contratados a partir de $1^{\circ}$ de janeiro de 1999; fixa as diretrizes de atenção à saúde; e revoga as Resoluções Normativas - RN no 387 , de 28 de outubro de 2015, e RN no 407, de 3 de junho de 2016. Brasília: Diário Oficial da União 2017; 8 nov.

42. Rodrigues DP, Alves VH, Penna LHP, Pereira AV, Branco MBLR, Souza MRP. O descumprimento da lei do acompanhante como agravo à saúde obstétrica. Texto \& Contexto Enfermagem 2017; 26(3):e5570015.

43. Narchi NZ, Castro CM, Oliveira CF, Tambellini F. Report on the midwives' experiences in the Brazilian National Health System: a qualitative research. Midwifery 2017; 53:96-102.

44. Brasil. Ministério da Saúde (MS). Gabinete do Ministro. Portaria no 11 , de 7 de janeiro de 2015. Redefine as diretrizes para implantação e habilitação de Centro de Parto Normal (CPN), no âmbito do Sistema Único de Saúde (SUS), para o atendimento à mulher e ao recém-nascido no momento do parto e do nascimento, em conformidade com o Componente PARTO E NASCIMENTO da Rede Cegonha, e dispõe sobre os respectivos incentivos financeiros de investimento, custeio e custeio mensal. Brasília: Diário Oficial da União 2015; 8 jan.

45. Leão MRC, Bastos MAR. Doulas apoiando mulheres durante o trabalho de parto: experiência do Hospital Sofia Feldman. Rev Lat Am Enfermagem 2001; 9(3):90-94

46. Vital M. Hospital da Mulher é o primeiro do Brasil na contratação de doulas pelo SUS. Agência Alagoas 2019 Out 14. [acessado 2020 Abr 24] Disponível em: http:// agenciaalagoas.al.gov.br/noticia/item/31285-hospital-da-mulher-e-o-primeiro-do-brasil-na-contratacao-de-doulas-pelo-sus

47. Cidade de São Paulo. Lei no 16.602, de 23 de dezembro de 2016. Dispõe sobre a permissão da presença de doula durante todo o período de trabalho de parto, parto e pós-parto imediato, bem como nas consultas e exames de pré-natal, sempre que solicitado pela parturiente, nas maternidades, hospitais e demais equipamentos da rede municipal de saúde. São Paulo: Diário Oficial Cidade de São Paulo 2016; $24 \mathrm{dez}$.

48. Brasil. Câmara dos Deputados. Projeto de Lei n ${ }^{\circ}$ 8363/2017. Dispõe sobre o exercício profissional da atividade de doula e dá outras providências.

49. Equipe de Base Warmis Convergência das Culturas. Saúde da mulher imigrante [Internet]. [acessado 2020 Abr 24]. Disponível em: http://www.warmis.org/projetos/saude-da-mulher-migrante.html

50. Buekens P, Alger J, Bréart G, Cafferata ML, Harville E, Tomasso G. A call for action for COVID-19 surveillance and research during pregnancy. Lancet Glob Heal 2020; 8(7):e877-e878.
51. Oppenheim M. Pregnant women forced to give birth without support in hospital amid coronavirus outbreak. Independent $2020 \mathrm{Mar} 31$. [acessado $2020 \mathrm{Abr}$ 25]. Disponível em: https://www.independent.co.uk/ news/uk/home-news/coronavirus-pregnant-womenbirth-hospital-nhs-parents-advice-a9439391.html

52. Ribeiro A, Knoploch C. Coronavírus impõe distância a pai, avós e doulas, e grávidas temem um parto solitário. O Globo 2020 Mar 29. [acessado 2020 Abr 24]. Disponível em: https://oglobo.globo.com/sociedade/coronavirus/coronavirus-impoe-distancia -pai-avos-doulas-gravidas-temem-um-parto-solitario- 24335320

53. Trapani Júnior A, Vanhoni LR, Silveira SK, Marcolin AC. Childbirth, Puerperium and Abortion Care Protocol during the COVID-19 Pandemic. Rev Bras Ginecol e Obstet 2020; 42(6):349-3555.

54. Ravaldi C, Wilson A, Ricca V, Homer C, Vannacci, A. Pregnant women voice their concerns and birth expectations during the COVID-19 pandemic in Italy. Women and Birth 2020; no prelo.

Artigo apresentado em 14/06/2020

Aprovado em 17/12/2020

Versão final apresentada em 19/12/2020

Editores-chefes: Romeu Gomes, Antônio Augusto Moura da Silva 
